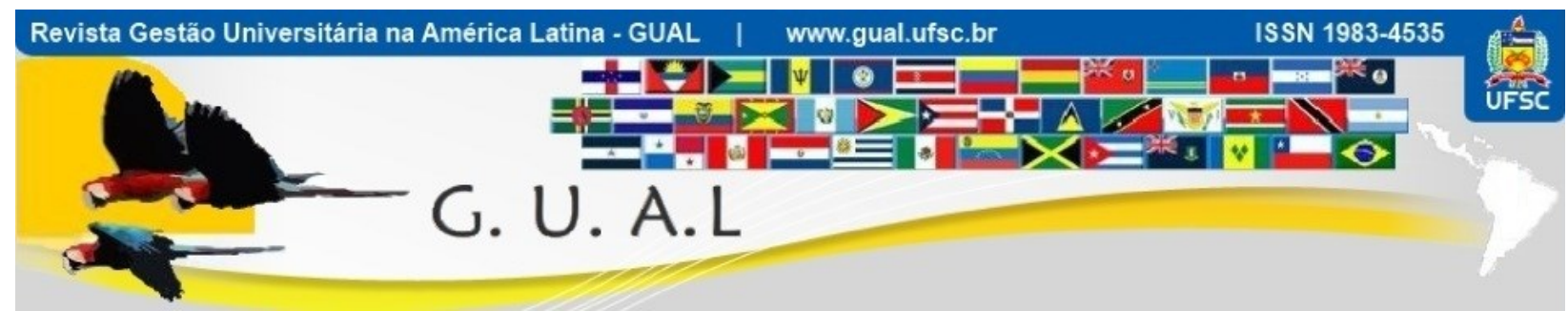

DOI: http://dx.doi.org/10.5007/1983-4535.2018v11n1p1

\title{
PROPOSTA DE MODELO PARA AVALIAÇÃO DA SATISFAÇÃO COM A QUALIDADE DO ENSINO
}

\section{PROPOSED MODEL FOR SATISFACTION EVALUATING WITH EDUCATIONAL QUALITY}

Rafaele Matte Wojahn, Doutoranda Universidade Regional de Blumenau - FURB rafaelewojahn@gmail.com

Sheila Patrícia Ramos, Doutoranda Universidade Regional de Blumenau - FURB spr80sc@gmail.com

Luciano Castro de Carvalho, Doutor Universidade Regional de Blumenau - FURB

luccar@gmail.com

Recebido em 07/junho/2016

Aprovado em 09/novembro/2017

Sistema de Avaliação: Double Blind Review

Esta obra está sob uma Licença Creative Commons Atribuição-Uso. 


\title{
RESUMO
}

Este estudo tem como objetivo apresentar uma proposta para avaliação da satisfação com a qualidade de serviços no ensino. As dimensões do modelo proposto foram baseados em estudos de Parasuraman et al (1985), com inserção de dimensões relacionadas ao Capital social (Coleman, 1988) e Didática (Libâneo 1994). Adotou-se neste estudo uma abordagem quantitativa. A população da pesquisa foram os estudantes dos níveis do Ensino Fundamental, Médio, Superior, Especialização e Pós-Graduação, sendo amostra composta por 458 estudantes. Os dados foram analisados por meio de Regressão Linear Múltipla. Os testes de confiabilidade do modelo apresentaram valores acima dos índices esperados mostrando-se um modelo competente para avaliar a satisfação da qualidade no ensino pelos discentes. A análise realizada mostrou que o modelo explica em $81 \%$ a satisfação geral dos discentes com a qualidade de ensino e também que, as dimensões relacionadas ao capital social de Coleman (1988), foram excluídas do modelo por não apresentarem significância. A dimensão com maior grau de explicação sobre a satisfação é a Didática, em 66,4\%.

Palavras-chave: Satisfação. Qualidade. Ensino.

\begin{abstract}
This study aims to present a proposal to assess the satisfaction with the service quality in teaching. The dimensions of the model were based on studies of Parasuraman et al (1985), Coleman (1988) and Libâneo (1994). It was adopted in this study a quantitative method. The research population was students of primary education, secondary education, higher education, specialization and graduate studies. The sample is composed by 458 students. Data were analyzed using multiple linear regressions. Model's reliability tests showed index above of expected, proving of being an efficient model to assess the satisfaction about service quality in teaching by students. However, the analysis carried out by means of multiple linear regression showed that the model explains $81 \%$ overall satisfaction about service quality in teaching and also that the dimensions related to social capital were excluded from the model due to the lack of significance. The dimension that most explain the satisfaction of students about service quality is the teaching method $(66,4 \%)$.
\end{abstract}

Keywords: Satisfaction. Quality. Education. 


\section{INTRODUÇÃO}

As Instituições de Ensino Superior (IES) não podem sentir-se excessivamente confiantes com a cota de mercado e as posições competitivas conquistadas, uma vez que estes podem não ser duradouros. O aumento da demanda e a entrada de novos competidores, como as universidades virtuais, exigem das IES constante atenção quanto à qualidade do serviço que oferecem (TORRES, 2004; MAINARDES, ALVES, RAPOSO, 2010).

O Ministério da Educação e Cultura (MEC) possui um indicador de qualidade de todos os cursos de graduação e pós-graduação de cada instituição de ensino. Esse indicador, denominado Índice Geral de Cursos (IGC), que varia de 1 a 5, em que 1 e 2 são considerados desempenho insatisfatório; 3, razoável; e 4 e 5, bom. No sítio do MEC constam a avaliação de 2.276 instituições de ensino, onde $1 \%$ obtiveram nota $5,14,9 \%$ nota $4,54,9 \%$ nota $3,16,56 \%$ nota $2,0,44 \%$ nota 1 e $12,2 \%$ sem conceito.

O interesse pela avaliação da educação superior no Brasil começou a se fortalecer no final da década de 70, quando os docentes começaram a organizar debates sobre o tema. Destaca-se, nesta mesma época, o início da avaliação realizada pela Coordenação de Aperfeiçoamento de Pessoal de Ensino Superior (CAPES) para credenciamento e recredenciamento de programas de pós-graduação; da avaliação dos pesquisadores; da avaliação da pesquisa produzida nas universidades realizada pelo Conselho Nacional de Desenvolvimento Cientifico (CNPq) e por outras instituições semelhantes; assim como, tentativas de auto avaliação de algumas universidades (DAVOK, 2008).

No Brasil, as instituições de educação superior vinculadas ao Sistema Nacional de Avaliação da Educação Superior (SINAES) devem cumprir as etapas de auto avaliação proposta pelo órgão. O SINAES foi criado pela Lei $\mathrm{n}^{\circ} 10.861$, de 14 de abril de 2004, e é formado por três componentes principais: a avaliação das instituições, dos cursos e do desempenho dos estudantes. Os resultados dessas avaliações possibilitam traçar um panorama da qualidade dos cursos e instituições de educação superior no Brasil. As informações obtidas com as avaliações são utilizadas pelas IES para orientação institucional, efetividade acadêmica e social; pelos órgãos governamentais para orientar políticas públicas e pelo público em geral, para orientar suas decisões quanto à qualidade dos cursos e das instituições (BRASIL, 2015).

Assim, a qualidade dos serviços oferecidos pelas IES se tornam relevantes para sua permanência no mercado e ainda, muitas vezes se torna fator instigante à busca pela liderança. 
A qualidade do ensino é, então, um fator que pode ser avaliado tanto pelo governo e seus órgãos competentes como pela própria IES e é objeto de inúmeros estudos acadêmicos que buscam criar instrumentos confiáveis e completos para tal avaliação.

No contexto da presente pesquisa, serviços são, segundo Berry e Parasuramam (1992, p. 20) dominados pela qualidade da experiência, atributos que só podem ser avaliados depois da compra e/ou durante o consumo. A maneira como o pessoal de serviço se comporta na presença de clientes - modo como agem, o que dizem, o que deixam de dizer, aparência influência o fato de os clientes voltarem a comprar da empresa.

Diante da relevância do exposto a realidade das IES, busca-se neste estudo apresentar uma proposta para avaliação da satisfação dos discentes com a qualidade no ensino. Desta forma, busca-se verificar se as dimensões utilizadas para medir a qualidade em serviço no ensino são percebidas pelos discentes da IES; e por meio de uma avaliação hierárquica de importância, verificar a dimensão da qualidade que possui maior impacto na satisfação dos discentes da IES com a qualidade do ensino.

Quanto à justificativa teórica, não foram encontrados artigos que compreenderam todos os construtos elencados neste, tais como dimensões relacionadas ao capital social e didática, ainda que há outros trabalhos relevantes que abordam a qualidade de serviço no ensino. Na forma prática, a presente pesquisa poderá contribuir também para a IES que poderá agir de forma estratégica visando melhorar seu desempenho e alcançar melhores resultados quanto à qualidade de seus serviços para seus alunos.

\section{REVISÃO BIBLIOGRÁFICA}

\subsection{Qualidade do Serviço no Ensino}

A liderança em serviços é o que propulsiona a melhoria em serviços e o que distingue uma empresa de seus concorrentes (BERRY, 1996).

Parasuramam et al (1985) identificaram 10 categorias, denominados de dimensões da qualidade. São elas: aspectos tangíveis, confiabilidade, presteza, competência, cortesia, credibilidade, segurança, acessibilidade, comunicação e compreensão/conhecimento do cliente. Posteriormente, Parasuraman et al (1988) complementaram seu modelo com um instrumento denominado SERVQUAL, composto por cinco dimensões gerais: Confiabilidade; Tangíveis; Presteza; Segurança e Empatia. 
Segundo Berry (1996) é preciso estabelecer um processo de pesquisa sobre qualidade em serviços que forneça informações oportunas e importantes para os gerentes considerarem na tomada de decisões. Fazer um estudo sobre a qualidade em serviços é análogo a se tirar um fotografia. O conhecimento profundo e o entendimento do padrão de mudança provém de uma série contínua de fotografias de vários assuntos em diferentes ângulos. O sistema de informações sobre qualidade em serviços permitem encorajar e habilitar a gerencia e incorporar a voz do cliente no processo de tomada de decisão; revelar prioridades; rastrear o desempenho em serviços; revelar o impacto das iniciativas e dos investimentos realizados na qualidade dos serviços; fornecer uma base de dados para recompensar os serviços de elevada qualidade e corrigir os de baixa qualidade.

Segundo Fortes, Silveira, Domingues e Souza (2011), entre os estudos que contribuem para o desenvolvimento de escalas para medir a qualidade de serviços apresentam-se, entre outros, Parasuraman; Berry; Zeithaml (1985); Parasuraman; Zeithaml; Berry (1988,1991a); Cronin; Taylor (1992); Stevens; Knuston; Patton (1995); Caruana; Pitt, (1997); Firdaus (2005); e Yildiz; Kara (2009). Figueredo, Tontini e Silveira (2006), além de Parasuraman; Berry; Zeithaml (1985), também destacam Kano et al. (1984) e Martilla e James (1977) representados pelo modelo Kano de qualidade atrativa e obrigatória, a matriz de importância e desempenho, respectivamente.

Souza, Silveira, Fortes e Domingues (2011) realizaram um estudo no qual buscaram comparar as dimensões apresentadas nos modelos de qualidade em serviços. Os modelos selecionados para este estudo foram: Qualidade dos Serviços (SERVQUAL), Importância da Qualidade dos Serviços (SERVQUAL Ponderado), Desempenho do Serviço (SERVPERF), Qualidade Interna (INTQUAL), Qualidade do Ensino Superior (HEDPERF), e Qualidade do Serviço Percebido no PESS (PESPERF). Seguindo o método de pesquisa definido, foi identificado, primeiramente, um elenco de 19 dimensões nos modelos estudados. A análise evidenciou que doze destas dimensões são utilizadas pela maioria dos modelos, de acordo com as características comuns. As doze dimensões propostas foram apresentadas, segundo a ordem de frequência: Confiabilidade; Lealdade; Segurança; Acesso; Conhecer/Compreender o cliente; Tangíveis; Competência; Comunicação; Cortesia; Receptividade; Aspectos acadêmicos e Aspectos não acadêmicos.

Souza, Silveira, Fortes e Domingues (2011) afirmam que estas dimensões formam uma base para a realização de medida de avaliação da qualidade de serviços e servem de base para amparar estratégias de avaliação em organizações educacionais de ensino superior. 
Segundo Berry (1996) avaliar a importância de atributos de serviço não é a mesma coisa que avaliar as expectativas de serviços dos clientes, embora sejam intimamente relacionadas. As expectativas dos clientes são padrões de comparação que usam para julgar o desempenho de vários atributos de serviço. Os clientes avaliam o desempenho em serviço segundo dois padrões: entre o serviço desejado e o serviço adequado. Contudo, os atributos de serviço não são uniformemente importantes para os clientes e, por isso, é útil se avaliar especificamente a importância relativa de cada um. Uma abordagem para se avaliar a importância de um atributo é solicitar aos entrevistados ou respondentes que os classifique por ordem de relevância, e ainda, alocar uma pontuação para as dimensões, com base na importância de cada um (BERRY, 1996).

Walter, Tontini e Domingues (2005) realizaram uma pesquisa cujo objetivo foi mostrar os resultados parciais de uma pesquisa acerca da satisfação de acadêmicos do curso de Administração, utilizando o Modelo Kano de Qualidade Atrativa e Obrigatória e outros métodos para análise na identificação de oportunidades de melhoria em Instituições de Ensino Superior (IES). Foram pesquisados 20 atributos do curso de administração, analisados por meio de uma pesquisa quantitativa, com uma amostra de 362 alunos das diferentes habilitações, turnos e fases. Estes 20 atributos foram agrupados em 6 categorias, são elas: atendimento, capacitação dos professores, métodos de ensino, atitude, conteúdo, infraestrutura. Neste estudo, a análise realizada demonstrou que o maior grau de satisfação acontece no segundo semestre, sendo os atributos, diversidade de método de ensino e integração de teoria e prática destaques. Já na análise da Matriz de Importância e Desempenho revelou como pontos fortes os atributos relacionamento com os professores, contribuição do curso para o desempenho profissional e biblioteca. Observa-se no entanto que neste estudo não foi realizada uma analise de confiabilidade das categorias estudas.

Assim, a didática ou método de ensino, também mostrou ser um quesito a ser avaliado no contexto do ensino quando analisada a satisfação com a qualidade no ensino. Segundo Libâneo (1994) a didática investiga as condições e formas que vigoram no ensino, e ao mesmo tempo, fatores condicionantes das relações entre a docência e a aprendizagem. Conforme Libâneo (1994, p. 53) técnicas, recursos e meios ou meios de ensino são complementos da metodologia, colocados a disposição do professor para enriquecimento do processo de ensino.

Outro fator relevante foi o relacionamento com os professores. No que tange ao quesito relacionamento reporta-se a Coleman (1988) e em sua teoria do Capital Social, 
correspondente aos laços ou redes existentes entre os indivíduos, como a confiança, de modo a facilitar as ações. A escola trata-se de uma instituição em que pode-se acumular capital social, ocorrido por meio da conexão entre os indivíduos, impactando em melhores resultados (PARCEL; DUFUR, 1996; LAI; WONG; FENG, 2015). Por meio de uma pesquisa qualitativa sobre o papel do capital social relativo ao acesso ao ensino superior em Latinos, González, Stoner e Jovel (2003) observaram que alguns estudantes apontaram o relacionamento com os professores favorável, fazendo com que as chances de ingresso em universidades de elite aumentassem. No entanto, Parcel e Dufer (1996) encontraram evidências que ajustes entre o capital da escolar e familiar podem aperfeiçoar ou inibir o desempenho do aluno.

Ainda sobre o capital social, o estudo realizado por Kim e Kim (2012) investigou dois tipos de ambiente escolar para a formação do capital social, denominados seletivo, focado em língua estrangeira e outro, não seletivo, sendo o ambiente escolar geral, identificando que estudantes do ambiente seletivo são mais propensos a formação de laços sociais quando comparado ao ambiente não seletivo. Entretanto, os resultados também indicaram que os laços de amizade formado entre os estudantes influenciam na vida social e acadêmica de forma significativa (KIM; KIM, 2012). Assim, o contexto escolar é um canal em que o capital social pode ser reforçado (LAI; WONG; FENG, 2015).

\section{METODOLOGIA}

No intuito de responder ao objetivo deste artigo adotou-se a abordagem quantitativa. A abordagem quantitativa refere-se a verificação entre variáveis de modo a testá-las observando uma teoria (CRESWELL, 2010).

Referente as técnicas de pesquisa, utilizou-se a descritiva, com estratégia survey ou de levantamento. A pesquisa descritiva objetiva medir e descrever características de uma questão de pesquisa (HAIR JR et al, 2005b). Conforme Creswell (2010), a pesquisa de levantamento estuda a percepção de uma amostra de uma população de forma numérica ou quantitativa. Quanto ao corte, foi o transversal, em que os dados foram coletados em um período de tempo específico (CRESWELL, 2010).

A população da pesquisa foram os estudantes variando entre os níveis Ensino Fundamental, Ensino Médio, Ensino Superior, Especialização e Pós-Graduação (mestrado ou doutorado). A amostra foi composta por 458 estudantes, classificada como amostragem por 
conveniência. A amostra por conveniência envolve a pesquisa com indivíduos acessíveis ao pesquisador e reduz o tempo na coleta de dados (HAIR JR et AL 2005b).

$\mathrm{O}$ instrumento de coleta de dados utilizado nesta pesquisa foi um questionário dividido em três blocos, em que foram mensuradas a percepção dos respondentes quanto aos fatores relacionados à satisfação e qualidade no ensino, por meio das seguintes dimensões Estrutura, Competência, Segurança, Cortesia, Acessibilidade, Relacionamento com os colegas, Relacionamento com os professores, Didática e Satisfação. No presente estudo, o modelo utilizado foi adaptado de Parasuramam et al. (1988), com as dimensões que influenciam a avaliação da qualidade de serviços e, Coleman (1999) sobre o capital social. E ainda incluído também a dimensão didática, considerada, devido ao contexto do estudo. As dimensões com a sua descrição e os autores podem ser observados no Quadro 01.

Quadro 01 Dimensões da pesquisa.

\begin{tabular}{|c|c|c|}
\hline Dimensão & Descrição & Referência \\
\hline $\begin{array}{l}\text { Aspectos } \\
\text { tangíveis }\end{array}$ & $\begin{array}{l}\text { Inclui a evidência física do serviço: } \\
\text { - Instalações físicas; } \\
\text { - Ferramentas e equipamentos utilizados para prestar o serviço; } \\
\text { - Representações físicas do serviço, etnre outros. }\end{array}$ & \multirow{5}{*}{$\begin{array}{c}\text { Parasuramam et al } \\
\qquad(1985)\end{array}$} \\
\hline Competência & $\begin{array}{l}\text { Posse das habilidades necessárias e conhecimento para executar } \\
\text { o serviço: } \\
\text { - Conhecimento e habilidade do pessoal de contato; } \\
\text { - Conhecimento e habilidade de pessoal de apoio operacional, } \\
\text { etnre outros. }\end{array}$ & \\
\hline Segurança & $\begin{array}{l}\text { É a ausencia do perigo, risco, ou dúvida: } \\
\text { - Segurança física; } \\
\text { - A segurança financeira; } \\
\text { - Confidencialidade. }\end{array}$ & \\
\hline Cortesia & $\begin{array}{l}\begin{array}{l}\text { Envolve educação, o respeito, consideração e } \\
\text { pessoal dimpatia do } \\
\text { operadoras de telefonia, etc.). }\end{array} \\
\text { (incluindo }\end{array}$ & \\
\hline Acessibilidade & $\begin{array}{l}\text { Acessibilidade e facilidade de contato: } \\
\text { - por telefone; } \\
\text { - internet; } \\
\text { - horário de atendimento adequado, entre outros. }\end{array}$ & \\
\hline $\begin{array}{l}\text { Relacionamento } \\
\text { com colegas }\end{array}$ & $\begin{array}{l}\text { O capital social advém de mudanças na relação entre indivíduos } \\
\text { de modo a facilitar ações. }\end{array}$ & \multirow[t]{2}{*}{ Coleman (1988) } \\
\hline $\begin{array}{l}\text { Relacionamento } \\
\text { com professores }\end{array}$ & $\begin{array}{l}\text { O capital social advém de mudanças na relação entre indivíduos } \\
\text { de modo a facilitar ações. }\end{array}$ & \\
\hline Didática & $\begin{array}{l}\text { Investiga as condições e formas que vigoram no ensino, e ao } \\
\text { mesmo tempo, fatores condicionantes das relações entre a } \\
\text { docência e a aprendizagem. }\end{array}$ & Libâneo (1994) \\
\hline
\end{tabular}

Fonte: elaborados pelos autores.

O primeiro bloco foi composto por 42 assertivas referente as dimensões que compõe o instrumento de coleta de dados, sendo: 8 para a Estrutura; 4 para a Competência; 5 para a Segurança; 3 para a Cortesia; 5 para a Acessibilidade; 3 para o Relacionamento com os 
colegas; 3 para o Relacionamento com os professores; 5 para a Didática, e; 6 para a Satisfação. As assertivas forma mensuradas por meio da Escala Likert de 7 pontos, variando entre 1 "discordo totalmente" e 7 "concordo totalmente".

O segundo bloco do questionário foi composto por uma questão, de ordenação das dimensões supracitadas por grau de importância, sendo o 1 "pouco importante" e 8 "muito importante".

Por fim, o terceiro bloco correspondeu a caracterização da amostra do respondente da pesquisa, com questões correspondentes a idade; gênero; estado em que reside; nível de estudo variando entre Pronatec, Ensino Médio, Ensino Técnico, Ensino Superior, Especialização, Pós-Graduação (mestrado ou doutorado) e Outros; tipo de instituição de ensino, variando entre Pública e Particular; Curso; indicação do semestre para universitários e da série para colegiais e; indicação do meio de transporte que o estudante utiliza para chegar a instituição de ensino, variando entre caminhando; ônibus intermunicipal; carro; bicicleta; ônibus municipal; lotação particular; moto e outros. O Quadro 02 apresenta as dimensões e as assertivas utilizadas neste estudo.

Quadro 02 Dimensões e assertivas da qualidade em serviço no ensino

\begin{tabular}{|c|c|c|}
\hline Dimensão & Questão & Assertiva \\
\hline \multirow{8}{*}{ Estrutura } & EST1 & $\begin{array}{l}\text { As instalações físicas (sala de aula, prédios, banheiros) são } \\
\text { adequadas ao uso. }\end{array}$ \\
\hline & EST2 & $\begin{array}{l}\text { Os ambientes (sala de aula, banheiro, pátio) da Escola/Universidade } \\
\text { estão sempre limpos. }\end{array}$ \\
\hline & EST3 & $\begin{array}{l}\text { A quantidade de livro na biblioteca é adequada à necessidade dos } \\
\text { alunos. }\end{array}$ \\
\hline & EST4 & O ambiente de estudo da biblioteca é agradável para uso. \\
\hline & EST5 & A cantina/restaurante atende a necessidade dos alunos. \\
\hline & EST6 & $\begin{array}{l}\text { Os laboratórios (informática, química, física, anatomia, etc.) são bem } \\
\text { equipados e modernos. }\end{array}$ \\
\hline & EST7 & Há vagas suficientes para os alunos no estacionamento. \\
\hline & EST8 & Os alunos possuem fácil acesso à Internet. \\
\hline \multirow{4}{*}{ Competência } & COM1 & Professores possuem formação adequada à disciplina ministrada. \\
\hline & COM2 & Os professores respondem rapidamente as solicitações dos alunos. \\
\hline & COM3 & A secretaria responde rapidamente as solicitações dos alunos. \\
\hline & COM4 & As disciplinas do curso estão relacionadas a realidade dos alunos. \\
\hline \multirow{5}{*}{ Segurança } & SEG1 & A área interna da Escola/Universidade é segura. \\
\hline & SEG2 & A área externa da Escola/Universidade é segura. \\
\hline & SEG3 & $\begin{array}{l}\text { Eu tenho certeza de que os meus dados (notas, cobranças } \\
\text { financeiras) não serão divulgadas para outras pessoas. }\end{array}$ \\
\hline & SEG4 & Os funcionários da Escola/Universidade são confiáveis. \\
\hline & SEG5 & A internet da Escola/Universidade é segura (proteção contra hacker) \\
\hline \multirow{3}{*}{ Cortesia } & COR1 & Os funcionários da Escola/Universidade são amigáveis. \\
\hline & COR2 & A direção da Escola/Universidade é amigável. \\
\hline & COR3 & Os professores da Escola/Universidade são amigáveis. \\
\hline
\end{tabular}




\begin{tabular}{|c|c|c|}
\hline Dimensão & Questão & Assertiva \\
\hline \multirow{5}{*}{ Acessibilidade } & ACE1 & $\begin{array}{l}\text { O site da Escola/Universidade possui informações importantes para } \\
\text { os alunos. }\end{array}$ \\
\hline & ACE2 & $\begin{array}{l}\text { Os funcionários da Escola/Universidade estão sempre disponíveis } \\
\text { para ajudar os alunos. }\end{array}$ \\
\hline & ACE3 & $\begin{array}{l}\text { Os professores da Escola/Universidade estão sempre disponíveis } \\
\text { para ajudar os alunos. }\end{array}$ \\
\hline & ACE4 & $\begin{array}{l}\text { O horário de atendimento da secretaria é adequado as necessidades } \\
\text { dos alunos. }\end{array}$ \\
\hline & ACE5 & $\begin{array}{l}\text { A Escola/Universidade possui vias de acesso facilitado (rampas e } \\
\text { piso tátil) para cadeirantes e deficientes visuais. }\end{array}$ \\
\hline \multirow{3}{*}{$\begin{array}{l}\text { Relacionamento } \\
\text { com os colegas }\end{array}$} & REC1 & $\begin{array}{l}\text { Tenho facilidade de trabalhar em grupos com os meus colegas de } \\
\text { classe. }\end{array}$ \\
\hline & REC2 & Tenho um bom relacionamento com meus colegas de classe. \\
\hline & REC3 & $\begin{array}{l}\text { Quando encontro dificuldade no ensino meus colegas de classe me } \\
\text { ajudam. }\end{array}$ \\
\hline \multirow{3}{*}{$\begin{array}{c}\text { Relacionamento } \\
\text { com os } \\
\text { professores }\end{array}$} & REP1 & Tenho liberdade para expressar minhas dúvidas com os professores. \\
\hline & REP2 & Percebo que os professores se importam com meu aprendizado. \\
\hline & REP3 & $\begin{array}{l}\text { O professor é flexível quanto ao uso do método de ensino para } \\
\text { atender as necessidades dos alunos. }\end{array}$ \\
\hline \multirow{5}{*}{ Didática } & DID1 & $\begin{array}{l}\text { As formas de avaliação do aprendizado (provas, trabalhos) são } \\
\text { adequadas ao curso. }\end{array}$ \\
\hline & DID2 & Os professores possuem métodos de ensino adequados. \\
\hline & DID3 & $\begin{array}{l}\text { Os professores fazem o uso de tecnologias para auxiliar na } \\
\text { aprendizagem. }\end{array}$ \\
\hline & DID4 & Os professores são criativos na apresentação das aulas. \\
\hline & DID5 & Os professores mantêm os alunos motivados durante o curso. \\
\hline \multirow{6}{*}{ Satisfação } & SAT1 & $\begin{array}{l}\text { De modo geral, estou satisfeito com aspectos da estrutura } \\
\text { (instalações físicas, bibliotecas, cantinas, estacionamento, sala de } \\
\text { aula, etc.) da Escola/Universidade. }\end{array}$ \\
\hline & SAT2 & $\begin{array}{l}\text { De modo geral, estou satisfeito com o tratamento recebido das } \\
\text { pessoas (funcionários e professores) da Escola/Universidade. }\end{array}$ \\
\hline & SAT3 & Eu me sinto seguro quando estou na Escola/Universidade. \\
\hline & SAT4 & De modo geral, estou satisfeito com o curso. \\
\hline & SAT5 & De modo geral, estou satisfeito com os professores. \\
\hline & SAT6 & O curso foi fornecido conforme prometido. \\
\hline \multirow{8}{*}{ Importância } & IMP1 & Estrutura \\
\hline & IMP2 & Competência \\
\hline & IMP3 & Segurança \\
\hline & IMP4 & Cortesia \\
\hline & IMP5 & Acessibilidade \\
\hline & IMP6 & Relacionamento com os colegas \\
\hline & IMP7 & Relacionamento com os professores \\
\hline & IMP8 & Didática \\
\hline
\end{tabular}

Fonte: Elaborado pelos autores com base em Parasuraman et al. (1985); Coleman (1988) e Libâneo (1994).

Para a coleta de dados optou-se em disponibilizar questionários por meio impresso e eletrônico (via Google Docs) de modo a facilitar a coleta e a captar diferentes perfis de estudantes. O período de coleta de dados ocorreu de dezembro de 2014 à maio de 2015. 
Os dados foram analisados pelo método estatístico Regressão Linear Múltipla, pelos modos enter e stepwise. A regressão consiste em verificar a relação existente entre uma variável dependente e várias independentes (HAIR JR et al, 2005). Já a regressão stepwise é utilizada quando deseja-se verificar estatisticamente o preditor entre as variáveis independentes (HAIR JR et al, 2005).

A confiabilidade foi calculada obedecendo a delimitação de cada dimensão. Utilizouse três métodos, sendo o Alpha de Cronbach (AC), a Confiabilidade Composta (CC) e a Variância Média Extraída (AVE). No Alpha de Cronbach refere-se à consistência interna do questionário em que valores mais próximos a 1 são considerados mais confiáveis (CRONBACH, 1951). A Confiabilidade Composta considera a variância de um construto em relação a variância total (ZAMBALDI; COSTA; PONCHIO, 2014). E, a Variância Total Extraída indica a confiabilidade por meio da variância nos indicadores, explicada pelo construto latente (HAIR JR et al, 2005). Para este artigo, são considerados os valores indicados pela literatura, sendo para o Alpha de Cronbach, 0,7; para a Confiabilidade Composta, 0,7, e; para a Variância Média Extraída, 0,5.

Para realização da Confiabilidade Composta e da Variância Média Extraída foi utilizado os escores apresentados pela Matriz de Componente dada pela Análise Fatorial Exploratória (AFE), utilizando 1 como o número de fatores, correspondente a dimensão em que os valores serão extraídos. Na próxima seção está descrita a análise dos dados.

\section{ANÁLISE DOS RESULTADOS}

A análise dos dados foi realizada em 6 etapas, correspondente caracterização da amostra da pesquisa, aos testes de confiabilidade, estatísticas descritivas e regressão linear múltipla pelos modos enter e stepwise e por fim, apresenta-se uma análise da hierarquia de importância das dimensões estudadas.

A primeira etapa da análise apresenta o perfil dos respondentes da pesquisa. Na Tabela 01 apresenta-se os resultados relativos a distribuição da amostra.

Conforme classificação de áreas do conhecimento apresentada pela CAPES. A organização das Áreas do Conhecimento apresenta uma hierarquização em quatro níveis, do mais geral ao mais específico, abrangendo nove grandes áreas nas quais se distribuem as 48 áreas de avaliação da CAPES. Estas áreas de avaliação, por sua vez, agrupam áreas básicas (ou áreas do conhecimento), subdivididas em subáreas e especialidades. A Tabela 01 
apresenta a distribuição da amostra da pesquisa nas grandes áreas do conhecimento conforme apresentadas pela CAPES.

Tabela 01 Distribuição da amostra.

\begin{tabular}{c|c}
\hline Grande área & Percentual da amostra \\
\hline Ciências Exatas e da Terra & $0,7 \%$ \\
\hline Linguística, Letras e Artes & $1,5 \%$ \\
\hline Ciências Agrárias & $2 \%$ \\
\hline Ciências da Saúde & $2,4 \%$ \\
\hline Ciências Humanas & $7,2 \%$ \\
\hline Engenharias & $11,1 \%$ \\
\hline Ciências Sociais e Aplicadas & $74,2 \%$ \\
\hline Outros & $0,9 \%$ \\
\hline Total & $100 \%$ \\
\hline
\end{tabular}

Fonte: Dados da pesquisa (2016).

Em relação ao gênero, a amostra é composta por 59,2\% do gênero feminino e 40,8\% do gênero masculino. Do total de 458 sujeitos que compõe a amostra, 87,3\% encontram-se cursando o ensino superior, $9,8 \%$ estão na pós-graduação e o restante, $2,9 \%$ estão distribuídos em especialização, ensino técnico, fundamental e Pronatec. No ensino superior, $48,5 \%$ da amostra encontram-se no período superior ao quinto semestre. Quanto à localização, 98\% afirmam que residem no Estado de Santa Catarina, 1,3\% no Estado de São Paulo, 0,4\% no Rio de Janeiro e 0,2\% no Estado do Rio Grande do Sul.

A segunda etapa da análise corresponde aos testes de confiabilidade, realizada pelos testes Alpha de Cronbach, Confiabilidade Composta e Variância Média Extraída. Os dados estão dispostos na Tabela 02:

Tabela 02 Teste de Confiabilidade

\begin{tabular}{|c|c|c|c|c|c|}
\hline Dimensão & Questão & $\begin{array}{l}\text { Matriz de } \\
\text { Componente }\end{array}$ & $\mathrm{AC}$ & $\mathbf{C C}$ & AVE \\
\hline \multirow{8}{*}{ Estrutura } & EST1 &, 702 & \multirow{8}{*}{0,779} & \multirow{8}{*}{0,92} & \multirow{8}{*}{0,48} \\
\hline & EST2 & ,691 & & & \\
\hline & EST3 & ,690 & & & \\
\hline & EST4 & 679 & & & \\
\hline & EST5 & 636 & & & \\
\hline & EST6 & 623 & & & \\
\hline & EST7 &, 529 & & & \\
\hline & EST8 &, 502 & & & \\
\hline \multirow{4}{*}{ Competência } & COM1 & 871 & \multirow{4}{*}{0,867} & \multirow{4}{*}{0,91} & \multirow{4}{*}{0,72} \\
\hline & COM2 & 848 & & & \\
\hline & COM3 & 842 & & & \\
\hline & COM4 & 824 & & & \\
\hline
\end{tabular}




\begin{tabular}{|c|c|c|c|c|c|}
\hline Dimensão & Questão & $\begin{array}{l}\text { Matriz de } \\
\text { Componente } \\
\end{array}$ & AC & $\mathrm{CC}$ & $\mathbf{A V E}$ \\
\hline \multirow{5}{*}{ Segurança } & SEG1 &, 829 & \multirow{5}{*}{0,853} & \multirow{5}{*}{0,92} & \multirow{5}{*}{0,64} \\
\hline & SEG2 & 801 & & & \\
\hline & SEG3 & ,792 & & & \\
\hline & SEG4 & ,787 & & & \\
\hline & SEG5 &, 767 & & & \\
\hline \multirow{3}{*}{ Cortesia } & COR1 & ,904 & \multirow{3}{*}{0,868} & \multirow{3}{*}{0,92} & \multirow{3}{*}{0,79} \\
\hline & COR2 & ,902 & & & \\
\hline & COR3 & ,863 & & & \\
\hline \multirow{5}{*}{ Acessibilidade } & ACE1 & ,874 & \multirow{5}{*}{0,842} & \multirow{5}{*}{0,92} & \multirow{5}{*}{0,67} \\
\hline & ACE2 & 857 & & & \\
\hline & ACE3 & ,779 & & & \\
\hline & ACE4 &, 753 & & & \\
\hline & ACE5 & ,693 & & & \\
\hline \multirow{3}{*}{$\begin{array}{l}\text { Relacionamento } \\
\text { com os colegas }\end{array}$} & REC1 & ,926 & \multirow{3}{*}{0,900} & \multirow{3}{*}{0,94} & \multirow{3}{*}{0,84} \\
\hline & REC2 & 915 & & & \\
\hline & REC3 & ,907 & & & \\
\hline \multirow{3}{*}{$\begin{array}{l}\text { Relacionamento } \\
\text { com os } \\
\text { professores }\end{array}$} & REP1 & ,863 & \multirow{3}{*}{0,876} & \multirow{3}{*}{0,92} & \multirow{3}{*}{0,80} \\
\hline & REP2 & ,921 & & & \\
\hline & REP3 & ,902 & & & \\
\hline \multirow{5}{*}{ Didática } & DID1 & 877 & \multirow{5}{*}{0,932} & \multirow{5}{*}{0,96} & \multirow{5}{*}{0,78} \\
\hline & DID2 & ,929 & & & \\
\hline & DID3 & ,835 & & & \\
\hline & DID4 & ,894 & & & \\
\hline & DID5 & ,904 & & & \\
\hline \multirow{6}{*}{ Satisfação } & SAT1 & 809 & \multirow{6}{*}{0,923} & \multirow{6}{*}{0,95} & \multirow{6}{*}{0,69} \\
\hline & SAT2 & ,838 & & & \\
\hline & SAT3 & ,779 & & & \\
\hline & SAT4 &, 895 & & & \\
\hline & SAT5 & ,895 & & & \\
\hline & SAT6 & 880 & & & \\
\hline
\end{tabular}

Fonte: Dados da pesquisa (2016).

Tabela 03 Análise Descritiva

\begin{tabular}{|c|c|c|c|c|c|c|c|c|c|c}
\hline \multirow{2}{*}{ Dimensão } & Questão & $\begin{array}{c}\mathbf{1} \\
\mathbf{( \% )}\end{array}$ & $\begin{array}{c}\mathbf{2} \\
\mathbf{( \% )}\end{array}$ & $\begin{array}{c}\mathbf{3} \\
\mathbf{( \% )}\end{array}$ & $\begin{array}{c}\mathbf{4} \\
\mathbf{( \% )}\end{array}$ & $\begin{array}{c}\mathbf{5} \\
(\mathbf{\%})\end{array}$ & $\begin{array}{c}\mathbf{6} \\
\mathbf{( \% )}\end{array}$ & $\begin{array}{c}\mathbf{7} \\
\mathbf{( \% )}\end{array}$ & Média & $\begin{array}{c}\text { Desvio } \\
\text { Padrão }\end{array}$ \\
\hline \multirow{5}{*}{ Estrutura } & EST1 & 3,1 & 6,8 & 10,9 & 17,7 & 27,7 & 22,1 & 11,8 & 4,7358 & 1,52946 \\
\cline { 2 - 12 } & EST2 & 2,6 & 4,4 & 11,4 & 16,4 & 21,6 & 25,5 & 18,1 & 4,9913 & 1,55425 \\
\cline { 2 - 11 } & EST3 & 3,3 & 4,1 & 5,7 & 10,7 & 16,6 & 24,5 & 35,2 & 5,4716 & 1,63263 \\
\cline { 2 - 11 } & EST4 & 1,3 & 2,4 & 3,3 & 6,1 & 11,4 & 28,4 & 47,2 & 5,9760 & 1,35635 \\
\cline { 2 - 11 } & EST5 & 11,6 & 7,6 & 10,9 & 19,9 & 19,9 & 16,8 & 13,3 & 4,3253 & 1,85144 \\
\cline { 2 - 10 } & EST6 & 8,7 & 8,5 & 12,7 & 21 & 20,5 & 19,7 & 9,0 & 4,3079 & 1,72102 \\
\cline { 2 - 10 } & EST7 & 41,3 & 15,5 & 11,8 & 12,2 & 8,1 & 5,9 & 5,2 & 2,6900 & 1,87927 \\
\cline { 2 - 10 } & EST8 & 28,8 & 17,2 & 14,4 & 14 & 9,6 & 9,0 & 7,0 & 3,1310 & 1,94010 \\
\hline
\end{tabular}




\begin{tabular}{|c|c|c|c|c|c|c|c|c|c|c|}
\hline Dimensão & Questão & $\begin{array}{r}1 \\
(\%) \\
\end{array}$ & $\begin{array}{r}2 \\
(\%) \\
\end{array}$ & $\begin{array}{r}3 \\
(\%) \\
\end{array}$ & $\begin{array}{r}4 \\
(\%) \\
\end{array}$ & $\begin{array}{r}5 \\
(\%) \\
\end{array}$ & $\begin{array}{r}6 \\
(\%) \\
\end{array}$ & $\begin{array}{r}7 \\
(\%) \\
\end{array}$ & Média & $\begin{array}{l}\text { Desvio } \\
\text { Padrão } \\
\end{array}$ \\
\hline \multirow{4}{*}{ Competência } & COM1 & 0,7 & 3,9 & 9,0 & 14,4 & 19,2 & 30,3 & 22,5 & 5,2860 & 1,44447 \\
\hline & COM2 & 2,2 & 3,5 & 10,7 & 17,9 & 20,5 & 28,8 & 16,4 & 5,0306 & 1,48926 \\
\hline & COM3 & 3,5 & 5,9 & 7,9 & 17,9 & 21,0 & 28,2 & 15,7 & 4,9432 & 1,57977 \\
\hline & COM4 & 1,7 & 7,9 & 8,1 & 15,3 & 22,9 & 29,3 & 14,8 & 4,9694 & 1,53413 \\
\hline \multirow{5}{*}{ Segurança } & SEG1 & 2,0 & 3,7 & 8,5 & 13,1 & 21,0 & 31,7 & 21,1 & 5,2271 & 1,48101 \\
\hline & SEG2 & 5,0 & 7,6 & 14,6 & 17,2 & 24,0 & 22,7 & 8,7 & 4,5066 & 1,60857 \\
\hline & SEG3 & 2,8 & 5,0 & 6,3 & 16,2 & 18,1 & 22,9 & 28,6 & 5,2489 & 1,62423 \\
\hline & SEG4 & 2,2 & 1,7 & 7,4 & 17,2 & 22,1 & 27,1 & 22,3 & 5,2555 & 1,44112 \\
\hline & SEG5 & 6,3 & 7,0 & 10,0 & 26,4 & 19,4 & 19,9 & 10,9 & 4,4891 & 1,63953 \\
\hline \multirow{3}{*}{ Cortesia } & COR1 & 1,7 & 3,7 & 7,6 & 17,5 & 20,5 & 26,4 & 22,5 & 5,2052 & 1,49009 \\
\hline & COR2 & 2,2 & 3,7 & 8,1 & 16,2 & 21,8 & 26,0 & 22,1 & 5,1790 & 1,51095 \\
\hline & COR3 & 1,5 & 3,5 & 4,4 & 13,8 & 19,2 & 35,4 & 22,3 & 5,4083 & 1,39760 \\
\hline \multirow{5}{*}{ Acessibilidade } & ACE1 & 1,5 & 1,1 & 4,4 & 7,0 & 15,1 & 32,5 & 38,4 & 5,8428 & 1,32034 \\
\hline & ACE2 & 1,1 & 4,8 & 5,5 & 18,8 & 21,4 & 29,9 & 18,6 & 5,1856 & 1,42287 \\
\hline & ACE3 & 2,0 & 5,7 & 5,9 & 14,2 & 21,6 & 29,7 & 21,0 & 5,2074 & 1,51672 \\
\hline & ACE4 & 4,4 & 6,6 & 4,8 & 14,2 & 17,7 & 26,6 & 25,8 & 5,1725 & 1,70170 \\
\hline & ACE5 & 6,3 & 8,7 & 10,0 & 16,6 & 15,5 & 23,4 & 19,4 & 4,7402 & 1,82139 \\
\hline \multirow{3}{*}{$\begin{array}{l}\text { Relacionamento } \\
\text { com os colegas }\end{array}$} & REC1 & 2,8 & 2,6 & 4,1 & 10,7 & 15,9 & 29,5 & 34,3 & 5,5983 & 1,50860 \\
\hline & REC2 & 0,7 & 2,6 & 3,3 & 7,6 & 15,1 & 32,8 & 38,0 & 5,8406 & 1,30087 \\
\hline & REC3 & 2,6 & 3,1 & 5,2 & 12,4 & 15,3 & 24,5 & 36,9 & 5,5568 & 1,56341 \\
\hline \multirow{3}{*}{$\begin{array}{c}\text { Relacionamento } \\
\text { com os } \\
\text { professores } \\
\end{array}$} & REP1 & 2,8 & 1,7 & 6,3 & 8,3 & 17,9 & 28,6 & 34,3 & 5,5961 & 1,50147 \\
\hline & REP2 & 5,5 & 4,8 & 7,4 & 12,0 & 22,7 & 22,7 & 24,9 & 5,0939 & 1,71552 \\
\hline & REP3 & 6,6 & 5,2 & 7,9 & 17,0 & 23,4 & 25,3 & 14,6 & 4,7991 & 1,67588 \\
\hline \multirow{5}{*}{ Didática } & DID1 & 3,9 & 5,5 & 5,0 & 16,8 & 25,1 & 27,3 & 16,4 & 5,0109 & 1,55634 \\
\hline & DID2 & 4,1 & 5,0 & 9,6 & 14,6 & 26,0 & 26,0 & 14,6 & 4,8974 & 1,57676 \\
\hline & DID3 & 3,1 & 4,4 & 8,7 & 15,1 & 21,4 & 26,9 & 20,5 & 5,1004 & 1,57064 \\
\hline & DID4 & 8,5 & 5,9 & 13,8 & 20,1 & 25,8 & 17,2 & 8,7 & 4,3537 & 1,65960 \\
\hline & DID5 & 8,7 & 8,7 & 15,1 & 18,6 & 21,4 & 17,9 & 9,6 & 4,2729 & 1,73576 \\
\hline \multirow{6}{*}{ Satisfação } & SAT1 & 4,4 & 6,8 & 10,9 & 16,6 & 26,9 & 21,8 & 12,7 & 4,7096 & 1,59895 \\
\hline & SAT2 & 2,8 & 3,3 & 9,0 & 13,3 & 23,8 & 28,6 & 19,2 & 5,1463 & 1,50942 \\
\hline & SAT3 & 2,4 & 4,8 & 6,6 & 10,5 & 21,8 & 32,8 & 21,2 & 5,2751 & 1,51241 \\
\hline & SAT4 & 2,8 & 6,6 & 10,9 & 11,8 & 17,9 & 28,6 & 21,4 & 5,0677 & 1,65577 \\
\hline & SAT5 & 6,3 & 6,1 & 8,7 & 13,8 & 20.5 & 28,6 & 15,9 & 4,8559 & 1,72032 \\
\hline & SAT6 & 4,1 & 7,2 & 10,0 & 14,4 & 15,5 & 28,8 & 19,9 & 4,9585 & 1,71632 \\
\hline
\end{tabular}

Fonte: Dados da pesquisa (2016).

A análise descritiva da Tabela 03 demonstrou por meio dos valores obtidos pela média que exceto para as assertivas de número 7 e 8 da dimensão Estrutura apresentaram valores abaixo da média considerando a escala utilizada $(3,5)$. Os escores apresentados indicam uma concordância dos respondentes em relação às assertivas constantes no questionário. Tais assertivas referem-se se há vagas suficientes no estacionamento da instituição e a facilidade de acesso na Internet, consecutivamente. 
Tabela 04 Descritiva de importância

\begin{tabular}{|c|c|c|c|c|c|c|c|c|c|c|c}
\hline Dimensão & Questão & $\mathbf{1}$ & $\mathbf{2}$ & $\mathbf{3}$ & $\mathbf{4}$ & $\mathbf{5}$ & $\mathbf{6}$ & $\mathbf{7}$ & $\mathbf{8}$ & Média & $\begin{array}{c}\text { Desvio } \\
\text { Padrão }\end{array}$ \\
\hline \multirow{5}{*}{ Importância } & IMP1 & 3,3 & 6,1 & 6,1 & 13,3 & 15,9 & 15,7 & 19,7 & 19,9 & 5,5764 & 1,96458 \\
\cline { 2 - 14 } & IMP2 & 4,1 & 2,8 & 1,3 & 2,4 & 2,2 & 5,5 & 12,2 & 69,4 & 7,0808 & 1,86175 \\
\cline { 2 - 13 } & IMP3 & 4,4 & 7,9 & 13,8 & 9,2 & 11,6 & 15,5 & 15,9 & 21,8 & 5,3515 & 2,16618 \\
\cline { 2 - 12 } & IMP4 & 20,7 & 6,3 & 7,2 & 7,9 & 10 & 13,1 & 15,9 & 18,8 & 4,7707 & 2,58379 \\
\cline { 2 - 12 } & IMP5 & 7,6 & 10,3 & 9,4 & 8,1 & 10,9 & 11,6 & 15,9 & 26,2 & 5,3384 & 2,36890 \\
\cline { 2 - 11 } & IMP6 & 5,7 & 7,4 & 7,9 & 10,9 & 14,2 & 12,4 & 17,7 & 23,8 & 5,4760 & 2,18120 \\
\cline { 2 - 11 } & IMP7 & 1,7 & 4,4 & 5,0 & 6,8 & 10,7 & 19,0 & 19,4 & 33,0 & 6,1987 & 1,85640 \\
\cline { 2 - 10 } & IMP8 & 4,1 & 3,3 & 2,6 & 3,3 & 3,9 & 10,0 & 22,9 & 49,8 & 6,7031 & 1,92395 \\
\hline
\end{tabular}

Fonte: Dados da pesquisa (2016).

De acordo com os resultados apresentados da Tabela 04, as variáveis seguem a seguinte ordem na escala de importância: Competência (IMP2), didática (IMP8), relacionamento com os professores (IMP7), acessibilidade (IMP5), relacionamento com os colegas (IMP6), segurança (IMP3) e estrutura (IMP1). Sendo a variável competência considerada mais importante e a variável estrutura menos importante na concepção da amostra.

A quarta etapa da análise será realizada por meio de Regressão Linear Múltipla. Foi utilizado como variável dependente a Satisfação e as demais dimensões, Estrutura, Competência, Segurança, Cortesia, Acessibilidade, Relacionamento com os professores, Relacionamento com os colegas e, Didática, relacionadas como variáveis independentes. Os resultados estão dispostos na Tabela 05:

Tabela 05 Regressão múltipla com todas as variáveis

\begin{tabular}{c|c|c|c|c}
\hline \multirow{2}{*}{$\begin{array}{c}\text { Resumo do } \\
\text { Modelo }\end{array}$} & $\mathbf{R}$ & $\mathbf{R}^{2}$ & \multicolumn{2}{|c}{$\mathbf{R}^{2}$ ajustado } \\
\cline { 2 - 5 } &, $904^{\mathbf{a}}$ &, 817 & \multicolumn{2}{|c}{, 814} \\
\hline Variáveis & $\mathbf{B}$ & Beta & SIG. & VIF \\
\hline (Constante) &,- 643 & &, 000 & 2,136 \\
\hline EST &, 297 &, 229 &, 000 & 3,615 \\
\hline COM &, 156 &, 144 &, 000 & 2,374 \\
\hline SEG &, 143 &, 128 &, 000 & 3,019 \\
\hline COR &, 103 &, 098 &, 006 & 3,657 \\
\hline ACE &, 109 &, 097 &, 012 & 3,521 \\
\hline REC &, 010 &, 010 &, 702 & 4,034 \\
\hline REP &, 062 &, 066 &, 079 & \\
\hline DID &, 285 &, 297 &, 000 & \\
\hline
\end{tabular}

a: Preditores: (Constante), DID, REC, EST, SEG, COR, REP, COM, ACE

Fonte: Dados da pesquisa (2016). 
O resumo do modelo indicou um índice de correlação (R) de 0,904, com coeficiente de determinação $\left(\mathrm{R}^{2}\right)$ de 0,817 , significando que as oito dimensões da qualidade explicam em $81 \%$ a satisfação geral.

No tocante as variáveis independentes, para refino do modelo foi observado a significância, cujo valores devem ser menores que 0,05 e, o VIF (Fator de Inflação da Variância) com valores abaixo de 5, de modo a selecionar as variáveis ausentes de multicolinearidade. Dada análise, as variáveis Relacionamento com os professores e Relacionamento com os colegas não apresentaram significância a nível de 0,05 resultado na eliminação da dimensão. Nenhuma dimensão foi retirada por não apresentar os valores de VIF maiores que o permitido. A Tabela 06 apresenta nova análise constando as variáveis de refino do modelo.

Tabela 06 Regressão múltipla com as variáveis significantes

\begin{tabular}{l|c|c|c|c}
\hline \multirow{2}{*}{$\begin{array}{l}\text { Resumo do } \\
\text { Modelo }\end{array}$} & $\mathbf{R}$ & $\mathbf{R}^{2}$ & \multicolumn{2}{|c}{$\mathbf{R}^{2}$ ajustado } \\
\cline { 2 - 5 } &, $903^{\mathrm{a}}$ &, 816 & \multicolumn{2}{|c}{, 814} \\
\hline Variáveis & $\mathbf{B}$ & Beta & SIG. & VIF \\
\hline (Constante) &,- 589 & &, 000 & 2,121 \\
\hline EST &, 292 &, 225 &, 000 & 3,560 \\
\hline COM &, 159 &, 148 &, 000 & 2,339 \\
\hline SEG &, 142 &, 128 &, 000 & 2,887 \\
\hline COR &, 117 &, 111 &, 001 & 3,543 \\
\hline ACE &, 122 &, 109 &, 004 & 2,920 \\
\hline DID &, 323 &, 337 &, 000 & \\
\hline
\end{tabular}

a: Preditores: (Constante), DID, EST, SEG, COR, ACE, COM

Fonte: Dados da pesquisa (2016).

Para a nova análise, encontrou-se a correlação entre as variáveis $(\mathrm{R})$ em 0,903 e o coeficiente de determinação $\left(\mathrm{R}^{2}\right)$ de 0,816 , indicando o poder de explicação das variáveis independentes em relação a dependente, satisfação, em $81 \%$. Os valores relativos a significância alteraram de 0,006 para 0,001 na dimensão Cortesia e 0,012 para 0,004 na dimensão Acessibilidade. Houve diminuição nos valores de VIF se comparando a Tabela 05 com a Tabela 06. 
Para prever o grau de Satisfação, a regressão linear múltipla proporcionou a seguinte equação:

$\mathrm{ESCORE}=-0,589+0,225(\mathrm{EST})+0,148(\mathrm{COM})+0,128(\mathrm{SEG})+0,111(\mathrm{COR})+0,109$ $(\mathrm{ACE})+0,337$ (DID)

Em que:

ESCORE: Satisfação

EST: Estrutura

COM: Competência dos professores e funcionários

SEG: Segurança

COR: Cortesia

DID: Didática

A quinta etapa da análise consistiu em realizar a regressão linear múltipla pelo método stepwise, buscando hierarquizar as dimensões da qualidade de acordo com o poder de explicação apresentado por cada uma. Os resultados encontram-se na Tabela 07.

Tabela 07 Regressão múltipla método stepwise

\begin{tabular}{c|c|c|c|c}
\hline Modelo & $\mathbf{R}$ & $\mathbf{R}^{2}$ & $\mathbf{R}^{2}$ ajustado & Sig. \\
\hline 1 &, $815^{\mathrm{a}}$ &, 664 &, 663 &, 000 \\
\hline 2 &, $873^{\mathrm{b}}$ &, 763 &, 762 &, 000 \\
\hline 3 &, $891^{\mathrm{c}}$ &, 793 &, 792 &, 000 \\
\hline 4 &, $897^{\mathrm{d}}$ &, 804 &, 802 &, 000 \\
\hline 5 &, $901^{\mathrm{e}}$ &, 812 &, 810 &, 000 \\
\hline 6 &, $903^{\mathrm{f}}$ &, 816 &, 814 &, 001 \\
\hline
\end{tabular}

apreditores: (Constante), DID

breditores: (Constante), DID, EST

'Preditores: (Constante), DID, EST, ACE

${ }^{\mathrm{d}}$ Preditores: (Constante), DID, EST, ACE, SEG

'Preditores: (Constante), DID, EST, ACE, SEG, COM

${ }^{f}$ Preditores: (Constante), DID, EST, ACE, SEG, COM, COR

Fonte: Dados da pesquisa (2016).

A análise da Tabela 07 demonstrou que a dimensão com maior grau de explicação sobre a Satisfação é a Didática, com coeficiente de determinação $\left(\mathrm{R}^{2}\right)$ de 0,664 e percentual de 66,4\%. A segunda dimensão que mais impacta sobre a Satisfação é a Estrutura com percentual de 9,9\% e grau de explicação do modelo acumulado em 76,3\%. As demais dimensões, Acessibilidade, Segurança, Competência e Cortesia juntas obtiveram o percentual de $5,3 \%$ e, percentual acumulado de $81,6 \%$ referente ao grau de explicação sobre a dimensão Satisfação. 
$\mathrm{Na}$ etapa seis, apresenta-se a análise da hierarquia de importância considerando importância relativa considerada pelos discentes e o resultado apresentado na regressão múltipla. Para verificar o que mais influencia na qualidade do ensino, os respondentes deveriam apontar, em uma escala de 1 a 8, o grau de importância para cada uma das dimensões que impactam na satisfação. Assim, foi realizada a análise de frequência para cada dimensão. Para a formação da hierarquia das dimensões foi considerado o posicionamento na escala em que a dimensão apontou maior frequência. Foi considerada a frequência para o ponto 8 da escala, já que exceto a dimensão Cortesia, as demais apresentaram maior frequência em tal posição. De modo a facilitar a análise, no Tabela 08 será apresentado as dimensões e a colocação da dimensão dada pela regressão múltipla e pelo apontamento do grau de importância, verificado pela frequência, pelos respondentes.

Tabela 08 Comparativo entre os fatores que influenciam na qualidade de ensino

\begin{tabular}{c|c|c|c}
\hline Dimensões & Estatística & Importância & Frequência \\
\hline Didática & $1^{\text {o }}$ & $2^{\text {o }}$ & 228 \\
\hline Estrutura & $2^{\circ}$ & $7^{\circ}$ & 91 \\
\hline Acessibilidade & $3^{\circ}$ & $4^{\circ}$ & 120 \\
\hline Segurança & $4^{\circ}$ & $6^{\circ}$ & 100 \\
\hline Competência & $5^{\circ}$ & $1^{\circ}$ & 318 \\
\hline Cortesia & $6^{\circ}$ & $8^{\circ}$ & 86 \\
\hline $\begin{array}{c}\text { Relacionamento com os } \\
\text { professores }\end{array}$ & $7^{\circ}$ & $3^{\circ}$ & 151 \\
\hline Relacionamento com os colegas & $8^{\circ}$ & $5^{\circ}$ & 109 \\
\hline
\end{tabular}

Fonte: Dados da pesquisa (2016).

A maior divergência apresentada comparando as colocações via regressão e frequência, foi na dimensão Estrutura, em $2^{\circ}$ e $7^{\circ}$ lugar, sucessivamente. Também apresentou divergência a dimensão Competência, em $5^{\circ}$ e $1^{\circ}$ lugar, sucessivamente. As dimensões Relacionamento com os professores ( $7^{\circ}$ e $3^{\text {a }}$ lugar) e, Relacionamento com colegas $\left(8^{\circ}\right.$ e $5^{\circ}$ lugar) não obtiveram significância estatística, conforme análise realizada anteriormente e disposta na Tabela 05, também apresentando discrepância em relação ao indicativo de importância para cada dimensão.

O ponto com maior frequência para a Cortesia foi o ponto 1, com valor de 95, e possuindo o rótulo de menor importância, ou seja, "Pouco importante". Desta forma, também foi verificada a frequência obtida para dimensão no ponto 8 da escala. $\mathrm{O}$ valor apresentado foi 86. A utilização da frequência do ponto 8 para esta dimensão não alterou a ordem da escala, 
ficando assim, todas as dimensões com a frequência apontada pelo ponto 8 da escala para a formação da hierarquia por grau de importância. O indicativo do grau de importância pelos respondentes para das dimensões apresentou resultados divergentes dos gerados pela análise estatística regressão múltipla pelo método stepwise. Neste caso, em primeiro lugar foi apontado a Competência, em segundo a Didática, em terceiro o Relacionamento com os professores, em quarto a Acessibilidade, em quinto o Relacionamento com os colegas, em sexto a Segurança, em sétimo a Estrutura e em oitavo a Cortesia.

\section{CONCLUSÕES}

Este estudo teve como objetivo apresentar uma proposta para avaliação da satisfação da qualidade no ensino. Para tanto foi elaborado um questionário a partir de dimensões baseadas em estudos como os de Parasuraman et al (1985), Coleman (1988) e Libâneo (1994).

Conforme análise dos dados, os testes de confiabilidade do modelo apresentaram valores acima dos índices esperados, exceto a Estrutura para a Variância Média Extraída, apresentando, no entanto, em outros dois testes de confiabilidade resultados considerados satisfatórios. Desta maneira, mostrando-se assim um modelo competente para avaliar a satisfação da qualidade no ensino pelos discentes.

Já na análise realizada por meio de Regressão Linear Múltipla, em que a satisfação foi considerada a variável dependente e as demais, Estrutura, Competência, Segurança, Cortesia, Acessibilidade, Relacionamento com os colegas, Relacionamento com os professores e Didática, variáveis independentes. O resultado desta análise revelou que as dimensões relacionadas ao capital social de Coleman (1988), relacionamento com os colegas e relacionamento com os professores, foram excluídas do modelo por não apresentarem significância em nível de 0,05. Em seguida, observou-se que o modelo explica em 81\% a satisfação geral dos discentes com a qualidade de ensino.

Buscando hierarquizar as dimensões do modelo realizou-se uma análise de Regressão Linear Múltipla, agora pelo método stepwise. Com isto observou-se que a dimensão com maior grau de explicação sobre a satisfação é a Didática, em 66,4\%. O modelo geral (excluído as dimensões Relacionamento com os colegas e Relacionamentos com os professores) obtevese um percentual acumulado de $81,6 \%$.

Comparando o resultado da hierarquia apresentada pela análise do modelo proposto por meio de Regressão Linear Múltipla pelo método stepwise com a hierarquização de 
importância atribuída pelos discentes da amostra (458 sujeitos) observou-se que a maior discrepância foi entre as dimensões Estrutura, $2^{\circ}$ (regressão) e $7^{\circ}$ lugar (importância atribuída pelos discentes), e Competência $5^{\circ}$ (regressão) e $1^{\circ}$ lugar (importância atribuída pelos discentes). Cabe destacar que a dimensão Didática encontrou-se em $1^{\circ}$ na regressão e $2^{\circ}$ na importância atribuída pelo discentes.

A dimensão Didática se mostrou relevante na avaliação do modelo proposto. Este quesito já havia sido apontado como relevante nos resultados encontrados nos estudos de Walter, Tontini e Domingues (2005). E foi reforçado neste estudo já que a Didática sozinha explica a satisfação geral dos discentes em $66,4 \%$. E na hierarquia encontra-se entre as primeiras posições, tanto na regressão como na importância atribuída pelos discentes, dentre as outras dimensões do modelo proposto.

No que se refere às dimensões do Capital Social de Coleman (1988), representadas no modelo pelas dimensões Relacionamento com os colegas e Relacionamento com os professores, estas não foram suportadas pelo modelo. Assim evidências apontadas por Coleman (1988) e Lai, Wong e Feng (2015) em que afirmam que o ambiente escolar permite o arranjo de conexões sociais ocasionando no acumulo de capital social, não foi confirmada por este estudo.

Assim, este estudo pretendeu contribuir para a pesquisa acadêmica no tocante aos modelos que atualmente são utilizados para avaliar a satisfação da qualidade no ensino. Convidamos outros pesquisadores a replicar e/ou ainda ampliar o modelo proposto que visam contribuir para a melhoria no ensino. 


\section{REFERÊNCIAS}

BONAMINO, Alicia et al. Os efeitos das diferentes formas de capital no desempenho escolar: um estudo à luz de Bourdieu e de Coleman. Revista Brasileira de Educação, v. 15, n. 45, p. 487-499, 2010.

BRASIL. Sinaes. Disponível em <http://portal.inep.gov.br/superior-sinaes>. Acesso 20 de abril de 2015.

COLEMAN, James S. Social capital in the creation of human capital. American Journal of Sociology, p. S95-S120, 1988.

CRESWELL, John W. Projeto de pesquisa: métodos qualitativo, quantitativo e misto. Artmed, 2010.

CRONBACH, Lee J. Coefficient alpha and the internal structure of tests. Psychometrika, v. 16, n. 3, p. 297-334, 1951.

DAVOK, Delsi Fries. Panorama histórico e metodologias de avaliação educacional. In:

Gestão de ensino superior: temas contemporâneos. Blumenau: Edifurb, 2008.

EBERLE, Luciene; MILAN, Gabriel Sperandio; LAZZARI, Fernanda. Identificação das dimensões da qualidade em serviços e a satisfação de clientes: um estudo aplicado em uma Instituição de Ensino Superior. RAE, São Paulo, v. 9, n. 2, 2010.

FIGUEREDO, Marcelo Salmeron; TONTINI, Gérson; SILVEIRA, Amélia. Integrando os modelos SERVQUAL, KANO e Matriz de Importância X Desempenho para avaliação de Instituições de Ensino Superior. In: Ensino na área de administração e avaliação em instituições de ensino superior. Blumenau, Edifurb, 2006.

FORTES, Victória Corrêa et al. Alternativas para a Avaliação Institucional em Instituição de Ensino Superior: Modelos SERVPERF e HEdPERF. EnEPQ, Paraíba, 2011.

GONZALEZ, Kenneth P.; STONER, Carla; JOVEL, Jennifer E. Examining the role of social capital in access to college for Latinas: Toward a college opportunity framework. Journal of Hispanic Higher Education, v. 2, n. 2, p. 146-170, 2003.

HAIR JR, Joseph F. et al. Análise multivariada de dados. Bookman, 2005a. 
HAIR JR, Joseph F. et al. Fundamentos de métodos de pesquisa em administração. 2005b.

KIM, Doo Hwan; KIM, Ji Hye. Emerging High-Status Track in South Korea: Social Capital Formation in the Social Contexts of Foreign Language and General High Schools. The AsiaPacific Education Researcher, v. 22, n. 1, p. 33-44, 2013.

LAI, Gina; WONG, Odalia; FENG, Xiaotian. Family, School, and Access to Social Capital Among High School Students in Urban Nanjing. American Behavioral Scientist, 2015.

LIBÂNEO, José Carlos. Didática. São Paulo, Cortez, 1994.

MAINARDES, Emerson Wagner; ALVES, Helena; RAPOSO, Mario. As instituições de ensino no século XXI: desafios para a gestão e relacionamentos com os stakeholders. In:

Reflexões sobre administração universitária e ensino superior. Curitiba: Juruá; Blumenau: Edifurb, 2010.

MAINARDES, Emerson Wagner; DOMINGUES, Maria José Carvalho de Souza; DESCHAMPS, Marcelo. Avaliação da qualidade nos serviços educacionais das instituições de ensino superior em Joinville/SC. REGE Revista de Gestão, v. 16, n. 1, p. 17-32, 2009.

MIGUEL, Paulo Auguto Cauchick; SALOMI, Gilberto Eid. Uma revisão dos modelos para medição da qualidade em serviços. Revista Produção, v. 14, n. 1, p. 12-30, 2004.

PARASURAMAN, Anantharanthan; ZEITHAML, Valarie A.; BERRY, Leonard L. A conceptual model of service quality and its implications for future research. The Journal of Marketing, p. 41-50, 1985.

PARASURAMAN, Anantharanthan; ZEITHAML, Valarie A.; BERRY, Leonard L. SERVQUAL: a multiple-item scale for measuring consumer perceptions of service quality. Journal of Retailing, v. 64, n. 1, p. 12 - 40, 1988.

PARCEL, Toby L.; DUFUR, Mikaela J. Capital at home and at school: Effects on student achievement. Social Forces, v. 79, n. 3, p. 881-911, 2001.

SOUSA, Tarcita Cabral Guisoni et al. Comparação de modelos de qualidade de serviços: proposição estratégica para instituições de ensino superior. Seme $\boldsymbol{A d}$, São Paulo, 2011. 
TORRES, Maria João Martins Saraiva. Função do marketing em Instituições de Ensino Superior. 119p. Dissertação (Mestrado em Design e Marketing) - Universidade do Minho, Guimarães, 2004.

WALTER, Silvana Anita; TONTINI, Gérson; DOMINGUES, Maria José Carvalho de Souza. Identificando oportunidades de melhoria em um curso superior através da análise da satisfação dos alunos. Encontro Nacional de Programas de Pós-Graduação em Administração, v. 29, 2005.

ZAMBALDI, Felipe; COSTA, Francisco José; PONCHIO, Mateus Canniatti; Mensuração em marketing: Estado atual, recomendações e desafios. Revista Brasileira de Marketing, v. 13, n. 2, p. 1-27, 2014. 